\title{
Reduktionismus und Rückübertragung
}

\author{
Patrick Grüneberg
}

»Welche [Zugangsweise] man wählt, hängt weitgehend von der Fragestellung ab und davon, was man eigentlich erreichen möchte. Jede Betrachtung, die sich nur auf den einen Zugang beschränkt, ist einseitig. Es gibt hier ein Spannungsfeld, das es auszuhalten gilt « ${ }^{1}$, so Ulrich Freund in einer Diskussion über das Verhältnis reduktionistischer und holistischer Verfahrensweisen in der Medizin. Während seine Äußerung durchaus von einem gewissen Optimismus geprägt ist, dass die Gegensätze zwischen Reduktionismus und Holismus nicht überbetont werden sollten, verdeckt sie doch ein grundlegendes Problem. Dieses Problem besteht darin, dass es, wenn erst eine Reduktion vollzogen wurde, äußerst schwierig ist, die Ergebnisse der Reduktion wieder zu rekontextualisieren. Die Schwierigkeit besteht darin, die bereichsspezifischen Ergebnisse in ihrer Bedeutung für den umfassenden Kontext, aus dem sie gewonnen wurden, zu interpretieren.

Generell gilt zunächst, dass Wissenschaft ohne Reduktion nicht vorstellbar ist, insofern eine je fachspezifische Reduktion den Untersuchungsgegenstand einer Disziplin konstituiert. Im Falle biologischer Modelle wird der Mensch auf seine biologischen Grundlagen reduziert. Während eine solche Reduktion also konstitutiv für einen disziplinären Zugang ist, ergeben sich Probleme, wenn die spezifischen Erkenntnisse wieder in den Gesamtzusammenhang, aus dem der je disziplinäre Untersuchungsgegenstand gewonnen wurde, rückübertragen werden. Dabei passiert es schnell, dass die zugangsspezifischen Resultate vorschnell verallgemeinert werden. Das führt dann dazu, dass der Mensch beispielsweise ausschließlich als ein biologisches Wesen betrachtet wird.

$1 \quad$ Freund in Deppert u. a. 1992, S. 38. 
Unmittelbare und nicht unbedingt erwünschte Folgen stellen sich dann beispielsweise in Gestalt evolutionärer Konzeptionen menschlicher Erkenntnis oder gesellschaftlicher Verhältnisse ein, die dann ausschließlich das Resultat biologisch fundierter Selektionsprozesse bilden. Aber auch die Biologie ihrerseits kann Gegenstand einer Reduktion werden. Physikalistisch basierte Ansätze benennen als ihren exklusiven Gegenstandsbereich die in der Physik gültigen Elemente der Wirklichkeit und reduzieren alle anderen Phänomene auf diese Elemente. Es sei hier programmatisch an das Sellarsche Diktum erinnert: »[I]n the dimension of describing and explaining the world, science is the measure of all things, of what is that it is, and of what is not that it is not. ${ }^{2}$ Die Frage, inwiefern die Biologie nun auch Teil der Naturwissenschaften in einem engeren Sinne ist oder doch im Unterschied zu den physikalistisch begründeten Wissenschaften einen eigenen Gegenstandsbereich umfasst, stellt eine komplizierte Aufgabe dar, da derlei Grenzen nur unter vielschichtigen Voraussetzungen zu ziehen sind. ${ }^{3}$

Die problematischen Folgen bereichsspezifischer Reduktionen zeigen sich erst dann, wenn eine Übertragung auf andere Bereiche stattfindet. Gleichzeitig ist eine solche Übertragung aber erwünscht, wenn die Erkenntnisse beispielsweise der Biologie auch in anderen wissenschaftlichen und Lebensbereichen anwendbar sein sollen. In methodischer Perspektive heißt das, dass Reduktionen auf eine reflektierte Weise eingesetzt werden müssen: Nach der spezifischen, für eine Disziplin konstitutiven Reduktion der Wirklichkeit bzw. bestimmter Aspekte derselben auf ihren methodisch bedingten Gegenstandsbereich und entsprechende Untersuchungsgegenstände muss bei der Rückübertragung der so gewonnenen Resultate der spezifische Charakter der Reduktion reflektiert werden, um den für den Gesamtkontext relevanten Aussagegehalt der spezifischen Fachkenntnisse zu ermitteln. Mit Blick auf biologische Forschung, insbesondere die Pharmazie, entstehen hinsichtlich der konkreten pharmazeutischen Produkte noch weiterführende Aspekte, die die Anwendbarkeit betreffen. Mit pharmazeutischen Präparaten werden den Konsumenten Mittel und Methoden an die Hand gegeben, die teils mit gravierenden Eingriffen in den Organismus und damit in den Lebenskontext einhergehen. Während eine bestimmte Wirkung also pharmazeutisch möglich und in Bezug auf bestimmte Symptome auch erwünscht ist, heißt das noch nicht, dass ein solches Präparat auch zugleich für den gesellschaftlichen Anwendungskontext geeignet ist. Die Rückübertragung reduktiv gewonnener Resultate erfordert daher zunächst ein Verständnis der zugrundeliegenden Reduktion.

Im Rahmen der folgenden Überlegungen möchte ich der Frage nachgehen, in welchem Verhältnis der nicht-reduzierte Mensch, also der Mensch im Angesicht der Komplexität und Vielfalt seiner Lebenswelt, zu den Bestimmungen einer reduktiven, und zwar der lebenswissenschaftlichen Beschreibungsebene seiner selbst steht. Dieses Verhältnis lässt sich insbesondere anhand des Problems der Rückübertragung lebenswis-

Sellars 1963, S. 173.

3 Vgl. zum programmatischen Verhältnis physikalischer und biologischer Wissenschaften Vollmer 2000 und Henning 2009. 
senschaftlicher Forschungsresultate in den lebensweltlichen Anwendungskontext verdeutlichen: Wie wird von der reduktiven Beschreibungsebene der Bezug zum "ganzen« Menschen wiederhergestellt? Diese Frage mitsamt dem (bio)ethischen Rattenschwanz stellt sich explizit im Kontext der Doping- und Enhancement-Problematik, aber auch schon grundlegender in der medizinischen Anwendung pharmazeutischer Präparate. Ethische Implikationen einer solchen biologischen Modellierung des Menschen zeigen sich dann vor allem im Kontext der Arzneimittelzulassung, da die Frage der Bewertung der Risiken sowie der Kosten vor allem auf lebenswissenschaftlicher Ebene thematisiert wird.

Im ersten Abschnitt steht die Reduktion des lebensweltlich situierten Menschen auf molekular basierte biochemische Reaktionen zentral. Die Unterscheidung zwischen einer deskriptiven und instrumentellen Reduktion im zweiten Abschnitt verdeutlicht die methodologischen Grundlagen einer verantwortungsvollen Rückübertragung. Die entscheidende Frage der Rückübertragung untersuche ich im dritten Abschnitt anhand der Systembiologie und der Arzneimittelzulassung. Im vierten und letzten Abschnitt differenziere ich die optimistische Aussicht Freunds dahingehend, dass die ethischen Implikationen biologischer Modelle durchaus lebenswissenschaftlich und in einem umfassenderen als nur einem Nutzen- und Effizienzdenken verpflichteten Kontext reflektiert werden könnten, wenn der politische bzw. öffentliche Wille vorhanden wäre.

\section{Konstitutive Reduktionen}

Ganz allgemein impliziert jede Wissensbestimmung die Negation anderer Wissensbestimmungen (determinatio negatio est), d. h., auch die spezifisch disziplinäre Bestimmung eines Untersuchungsgegenstandes geht zu Lasten anderer Bestimmungen derselben Ausgangsmenge. Wird der Mensch in einer biologisch-medizinischen Perspektive thematisiert, wird beispielsweise von sozialen und kulturellen Bestimmungen abgesehen (wobei soziobiologische Ansätze als Versuche gelten können, bereichsspezifische Erklärungen auch auf andere, zuvor ausgeschlossene Bereiche anzuwenden). Der zumindest hypothetische Ausgangspunkt wäre in diesem Fall der nicht biologisch reduzierte, der nicht-modellierte Mensch. ${ }^{4}$ Weiterhin konkretisiert sich die biologischmedizinische Untersuchung in der Abgrenzung je spezifischer funktionaler Subsysteme gemäß der medizinischen Fachgebiete oder chemisch-physikalischer Reaktionssysteme organischer Strukturen. Biologische Vorgänge in Zellen sollen in der Biochemie ihrerseits auch wieder reduziert werden, und zwar auf solche Prozesse, die sich mittels der organischen Chemie beschreiben lassen usw.

4 Ohne diesen epistemologischen Aspekt hier näher ausführen zu können, kommt dem Begriff des nicht-modellierten Menschen lediglich eine regulative Funktion zu, da jeder wissenschaftliche Ausgriff auf den lebensweltlich situierten Menschen immer eine Modellierung impliziert. Die eigentliche relevante Frage zielt dann auf die Variablen einer Modellierung ab, jedoch nicht auf die Modellierung selbst. 
Eine Reduktion versteht sich zumeist als eine intertheoretische Relation, d. h., es sollen beispielsweise biologische auf physikalische Erklärungen zurückgeführt werden. Oder physiologische Vorgänge auf biochemische und diese wiederum auf molekulare Prozesse. Das Ziel einer solchen Reduktion liegt in einer Vereinfachung bzw. Vereinheitlichung von Theorien, die allerdings durchaus als zweifelhaft zu beurteilen ist, weil mit der Etablierung einer zugrundeliegenden Mikroebene nicht per se eine Vereinfachung einhergeht. Gerade das klassische Beispiel einer physikalistischen Reduktion zeigt, dass die Ebene der Elementarteilchen nicht zwangsläufig zu einem übersichtlichen und eindeutig zu bestimmenden Set von Basisentitäten führen muss. Ein deutliches Beispiel aus dem physikalischen Bereich bildet hier die mathematische Beschreibung des Lasers, die mit der schwerlich fassbaren Menge von $10^{14}$ nicht-linearen Differentialgleichungen operiert. ${ }^{5}$

Methodologisch gesehen gliedert sich das Feld des Reduktionismus in zwei Varianten. ${ }^{6}$ In ontologischer Hinsicht gilt, dass alles, was auf einer Makroebene existiert, aus Teilen einer Mikroebene zusammengesetzt ist. Diese Form ist die harmlosere, weil sie unmittelbar keine forschungspraktischen Konsequenzen nach sich zieht. Vielmehr kommt in ihr eine naturwissenschaftliche Grundhaltung zum Ausdruck. Im Sinne eines pragmatisch eingeschränkten Reduktionismus kann man ontologisch gesehen Reduktionist sein und sich gleichwohl der explanatorischen Vielfalt anderer Wissenschaften bedienen. Die methodologische Reduktion geht dann ein entscheidendes Stück weiter, indem eine Theorie auf eine andere reduziert und damit ersetzt werden soll. Mit Blick auf die Biologie wäre hier an die biochemische Erklärung der zellulären Energiegewinnung und damit die Aufgabe vitalistischer Konzepte zu denken. Im Folgenden konzentriere ich mich auf diese methodologische Variante, wobei es mir letztlich nicht um eine intertheoretische, sondern um eine theoriekonstitutive Reduktion geht, d. h. die methodologische Voraussetzung dafür, dass ein spezifischer Gegenstand(sbereich) überhaupt als ein solcher definiert und damit erforscht werden kann. Somit lässt sich das Verfahren der Reduktion nicht nur in einem pejorativen Sinne charakterisieren: Wissenschaft ist ohne Reduktion nicht vorstellbar, weil eine je spezifische Reduktion den Untersuchungsgegenstand einer Disziplin konstituiert. Im Falle biologischer bzw. medizinischer Theorien wird der Mensch auf seine biologischen Grundlagen reduziert. Um der heutigen Spezialisierung in den Naturwissenschaften gerecht zu werden, müsste man das weite Feld der Biologie bzw. umfassender der Lebenswissenschaften auch wiederum genauer aufschlüsseln, weil auch zwischen den Lebenswissenschaften intertheoretische Reduktionen stattfinden. In theoriekonstitutiver Hinsicht werde ich von den intertheoretischen Verflechtungen in den Lebenswissenschaften abstrahieren, um der Lebenswissenschaft insgesamt den Menschen in seinem sozial-kulturellen Umfeld gegenüberzustellen. Somit können als Reduktandum die sozial-kulturell eingebundenen Menschen und als Reduktans bzw. als Untersuchungsgegenstand lebenswissen-

5 Vgl. Stöckler 1992, S. 169 f.

6 Vgl. Wolters 1996, ausführlicher Hoyningen-Huene - Wuketits 1989. 
schaftlicher Theorien biochemische Prozesse angegeben werden. ${ }^{7}$ Letztere lassen sich auf molekulare Prozesse eingrenzen, insofern die molekulare Medizin als aktuell vorherrschendes Paradigma angesehen werden kann, sofern es sich um therapeutische Eingriffe am Menschen handelt. ${ }^{8}$ Auch wenn die Formel des "sozial-kulturell eingebundenen Menschen" sehr offen ist, eignet sie sich dennoch als kritischer Gegenbegriff zur natur- bzw. lebenswissenschaftlichen Forschung, insofern sie im Verhältnis zu den Natur- und Lebenswissenschaften einen Grundbegriff bildet. Menschen in sozial- und kulturwissenschaftlicher Perspektive unterliegen natürlich auch wieder entsprechenden bereichsspezifischen Reduktionen. Daher ist die genannte Formel mehr im Sinne eines vortheoretischen Grundbegriffes zu verstehen.

\section{Deskriptive und instrumentelle Reduktion}

Während eine Reduktion also zunächst einen disziplinären Zugang konstituiert, ergeben sich meist Probleme, wenn die spezifischen Erkenntnisse wieder in den Gesamtzusammenhang, aus dem der je disziplinäre Untersuchungsgegenstand gewonnen wurde, rückübertragen werden. Dabei droht die Gefahr, dass die zugangsspezifischen Resultate vorschnell mit der folgenschweren Konsequenz verallgemeinert werden, dass der Mensch beispielsweise ausschließlich als ein biologisches Wesen betrachtet wird. Folgen sind evolutionäre Konzeptionen menschlicher Erkenntnis oder gesellschaftlicher Verhältnisse, die dann lediglich das Resultat biologisch fundierter Selektionsprozesse bilden. Die methodologisch entscheidende Einschränkung liegt darin, dass diese Konzeptionen sich dann selbst evolutionsbiologisch ableiten müssen, was auf einen bösartigen Zirkel hinausläuft. ${ }^{9}$ Ein weiteres generelles Problem intertheoretischer Reduktionen liegt in qualitativen Sprüngen zwischen verschiedenen Ebenen der Wirklichkeit, so dass sich beispielsweise molekulare nicht hinreichend mittels atomarer Prozesse erklären lassen. Mit Blick auf die Lebenswissenschaften ist auf die Medikalisierung des Menschen zu verweisen, die das Resultat einer Hypostasierung einer biochemischen bzw. molekularen Grundlegung des Menschen bildet und in dieser Absolutsetzung zu weitreichenden Problemen auf der Ebene des sozial-kulturellen Gesamtkontextes des Menschen führt. Stichworte wären hier Medikamentenabhängigkeit oder die heftig diskutierte Kostenentwicklung im Gesundheitswesen in Relation zum teils unklaren Nutzen vieler Medikamente. ${ }^{10}$

7 Überlegungen zum Organismusbegriff beziehen sich auf die Grundlagen der Biologie mit dem Ziel, Lebewesen umfassender als nur im Sinne chemischer Reaktionseinheiten zu betrachten; vgl. dazu den Beitrag von Michelini in diesem Band. Auch das Individuum stellt im Angesicht der sozialen Eingebundenheit des Menschen nicht unbedingt die kleinste Einheit dar (vgl. zu den Schwierigkeiten des methodischen Individualismus den Beitrag von Asmuth).

8 Vgl. Heinemann 2007.

9 Vgl. Binkelmann in diesem Band zum Verhältnis von Moral und Evolutionsbiologie.

10 Vgl. Schneider u. a. 2011, Holzbach u. a. 2010 und Schumann 2011. 
Diese Probleme im anwendungspraktischen Horizont lebenswissenschaftlicher Produkte reflektieren eine zugrundeliegende Ambivalenz hinsichtlich des Untersuchungsgegenstandes. Einerseits werden molekulare Prozesse untersucht, von denen aber andererseits angenommen werden muss, dass sie das Reduktandum (den Menschen) hinreichend repräsentieren. Da die Reduktion aber gerade darauf beruht, lediglich bestimmte Merkmale des Reduktandums (hier die biochemische Konstitution des Menschen) zu berücksichtigen, ist gerade mit Blick auf den anwendungspraktischen Horizont unklar, worin letztlich das Objekt des Reduktans liegt: Sind es molekulare Prozesse oder doch der gesamtkontextualisierte Mensch? Es muss also unterschieden werden zwischen einem deskriptiven Gebrauch des Reduktans (Analyse molekularer Reaktionen) und einem instrumentellen Gebrauch mit Blick auf das Reduktandum, der im Sinne einer Zwecksetzung (z. B. Therapie oder Leistungssteigerung) normativ fundiert ist. ${ }^{11}$ Das Ziel der reduktiven Analyse ist in der Pharmazie die Synthese von Wirkstoffen und damit die Übertragung einer molekularen Reaktion (Wirkung) auf das Reduktandum. Eine verantwortliche Übertragung ist jedoch nur dann möglich, wenn weitere, nicht unmittelbar aus der molekularen Reaktion ableitbare Faktoren berücksichtigt werden, wie insbesondere die Bestimmung von Gebrauchsvorschriften aufgrund individueller Unterschiede zwischen den Konsumenten der Präparate. ${ }^{12}$

Lebenswissenschaftliche Disziplinen, die wie die Medizin oder Pharmazie in einem lebensweltlichen Anwendungskontext praktiziert werden, unterliegen damit einem doppelten Bezug auf das Reduktans und das Reduktandum: Während die explanatorische Absolutsetzung des Reduktans konstitutiv für die lebenswissenschaftliche Reduktion ist, darf das Reduktans - formaliter bzw. methodologisch - wiederum nur regulativ verwendet werden, d. h., es darf zwar davon ausgegangen werden, dass die molekulare Reduktion des Menschen notwendige Bedingungen menschlicher Existenz bestimmt, aber nicht, dass diese reduktive Repräsentation des Reduktandums hinreichend ist. Es muss also trotz der zwingend notwendigen Reduktion diese selbst auch wieder hinsichtlich des lebensweltlichen Anwendungshorizontes zwingend relativiert werden. Die konkreten Konsequenzen dieser methodologischen Einschränkung für die lebenswissenschaftliche Forschungspraxis, also die Vermeidung einer Äquivokation des Reduktans mit dem Reduktandum, möchte ich im Folgenden weiter untersuchen. Es stellt sich in zugespitzter Form die Frage, wie die molekulare Wirkungsgenese auf die normativen Zwecksetzungen hinsichtlich des Gesamtsystems Mensch rückbezogen werden kann.

11 Das unklare Verhältnis von Therapie und Leistungssteigerung bildet auch eine entscheidende Unzulänglichkeit in der Begründung des Dopingverbots; vgl. Grüneberg 2010.

12 Vgl. zum Stellenwert des Individuellen in der Pharmazie den Beitrag von Kut Bacs in diesem Band. 


\section{Versuch einer Rückübertragung der Resultate auf das Reduktandum}

Der erste Schritt biologischer Modellierung besteht in der molekularen Reduktion des Menschen. Die folgenden Überlegungen zur Rückübertragung dürfen vor diesem Hintergrund nicht als antireduktionistisch aufgefasst werden, weil die Reduktion zuvor als theoriekonstitutiver Schritt identifiziert wurde. Die besondere methodologische Herausforderung besteht also darin, wie im Angesicht der molekularen Reduktion des Menschen dennoch ein Bezug zum "ganzen« bzw. sozial-kulturell kontextualisierten Menschen hergestellt werden kann, um nicht nur die medizinische, sondern auch die lebensweltliche Relevanz medizinischer Produkte und Verfahren reflektieren und bewerten zu können.

In der Rückübertragung werde ich zwei Ebenen unterscheiden: Der nach der Reduktion zweite Schritt vollzieht sich auf der lebenswissenschaftlichen Ebene im Übergang vom Molekül zum biologischen Gesamtsystem Mensch, um im dritten Schritt auf der lebensweltlichen Ebene vom biologischen Gesamtsystem in den sozial-kulturellen Anwendungskontext zu gelangen. Es wird zu prüfen sein, inwiefern diese idealtypische Rückübertragung de facto, also mit den vorhandenen wissenschaftlichen Ressourcen möglich ist. Dabei kommt diesem Schema einer Rückübertragung selbst ein regulativer Status zu, weil sich die medizinische Praxis auch ohne eine methodologisch basierte Rückübertragung als Anwendungswissenschaft immer so verhalten muss, als ob der lebensweltliche Bezug gegeben sei. ${ }^{13}$

Auf der lebenswissenschaftlichen Ebene bzw. innerhalb des Schnittfeldes von Medizin und Biologie kann die Systembiologie als Ansatz einer reduktionsimmanenten Rückübertragung verstanden werden. ${ }^{14}$ Nach Hood handelt es sich dabei um das P4Paradigma: Die Systembiologie verfährt predictive, preventive, personalized und participatory $^{15}$ als ein interdisziplinärer und theoriebasierter Ansatz aus der Biologie (quantitative Analytik, Modellvorstellung, genetische Modifikation), der Informatik (Datenbanken, Modellierungswerkzeuge, Visualisierung) und den Systemwissenschaften (Analyse, Modellierungskonzept, Synthese) ${ }^{16}$. Das Ziel sind vor allem quantitative Beschreibungen nicht nur der (molekularen bzw. physikochemischen) Komponenten biologischer Systeme, sondern deren Interaktion. Komplexe biologische Vorgänge sollen durch eine mathematische Modellierung fassbar werden, um derart die Datenflut beispielsweise der Genomforschung überhaupt verwerten zu können. Die allgemeine Vorgehensweise besteht darin, hinsichtlich eines biologischen Phänomens experimentelle Daten über Strukturen, Funktionen und Interaktionen zu sammeln, die dann in ein mathematisches Modell eingehen. Damit kann eine Hypothese formuliert werden, die

13 Vgl. zur Medizin als praktische Wissenschaft Rager 2007.

14 Vgl. zur Systembiologie Cornish-Bowden 2006 und Trewavas 2006.

15 Vgl. Tian u. a. 2012 und Bringmann u. a. 2007, S. V.

16 Vgl. Forschungszentrum Jülich $\mathrm{GmbH} 2008$. 
sowohl mittels in silico-Experimenten (Simulation) als auch mittels in vivo-Experimenten getestet werden kann. Im Bestfall erhält man eine virtuelle Repräsentation von Zellen oder ganzen Organismen. Als methodologisch wichtiger Unterschied gegenüber einer physikochemischen Reduktion gilt die Einbeziehung der Zeit, so dass nicht nur räumliche Reaktionen von Molekülen, sondern auch deren zeitliche Verteilung untersucht wird. Dieser Schritt erscheint insofern notwendig, weil es sich bei Organismen um komplexe Systeme handelt. Nach Rosen gilt ein System dann als komplex, wenn es nicht simulierbar ist. Das Scheitern einer Simulation wiederum beruht auf dem Problem des unendlichen Regelungsregresses ${ }^{17}$, das sich anhand von Nebenwirkungen darstellen lässt. Wie Folkers u. a. verdeutlichen, entsteht eine Nebenwirkung durch eine begrenzte Analyse molekularer Reaktionen bzw. der "multimodal action of a drug « ${ }^{18}$. Die Multimodalität unterläuft dabei die one-ligand one-protein-/one-target one diseaseKonzeption. Diese Konzeption beruht auf Paul Ehrlichs Konzept der sogenannten Zauberkugel und besagt, dass man einen Liganden entwickeln kann, der nur auf ein Protein präzise passt und eine Reaktion auslöst. Entgegen dieser Hypothese zeigt sich aber, dass ein Ligand aufgrund mehrerer Bindungsmöglichkeiten neben der erwünschten Reaktion auch noch diverse andere Reaktionen auslösen kann. Aber selbst wenn man auch der multimodalen Wirkung Rechnung tragen sollte, stellt sich das Problem, dass die Zielproteine in der notwendigen Konzentration nicht unbedingt zur selben Zeit am selben Ort sind, so dass erneut weitere Parameter das Design eines Wirkstoffes erschweren. Die Systembiologie zeigt, dass die molekularen Funktionen der Gene in einem physikochemischen Netzwerk inbegriffen sind und somit nicht von einer multimodalen Wirkung ausgegangen werden kann, ohne die zeitliche und räumliche Verteilung der Zielproteine zu berücksichtigen. Der unendliche Regelungsregress stellt sich hier also als das Erfordernis, immer neue Randbedingungen berücksichtigen zu müssen. Theoretisch müsste dieser Regress ein Ende haben, weil biologische Organismen eine lebensfähige Stabilität aufweisen; forschungspraktisch stellen sich die Organismen aber als sich stets erweiternde Kaskaden neuer Interaktionen und Randbedingungen dar, die in ihrer Gesamtheit - zumindest in absehbarer Zeit - nicht einholbar erscheinen.

Im Angesicht dieser strukturellen Problematik tritt die Systembiologie mit dem Anspruch einer auf den individuellen Patienten zugeschnittenen Bestimmung der biologischen Prozesse, die das lebendige Gesamtsystem konstituieren, auf. Die systemische Betrachtung steht dabei nicht in einem Ausschlussverhältnis zur molekularen bzw. physikochemischen Betrachtung. Während letztere von der biologischen Organisation abstrahiert, fokussiert die systemische Betrachtung auf die funktionelle Organisation des Systems. Ein Ausschluss ist deshalb nicht gegeben, sofern die Funktionalität des Gesamtsystems im Sinne einer Emergenz, also die Entstehung einer stabilen Zelle, stabiler Organe etc. aus der Interaktion der Zellbestandteile, erklärt werden soll. Der An-

17 Vgl. Rosen 1992, S. $268 \mathrm{f}$.

18 Folkers u. a. 2009, S. 8. 
spruch, das Gesamtsystem Mensch bzw. darüber hinausgehend das den Menschen umfassende Ökosystem zu beschreiben, fungiert aktuell nur als Zielsetzung und scheint vor dem Hintergrund, dass das Problem "how new properties emerge from interactions recurs at various levels of biological organization « ${ }^{19}$, also von der Ebene der Ribosomen, die für die Proteinsynthese zuständig sind, bis hin zu der Etablierung eines stabilen Ökosystems, noch in eher unerreichbarer Ferne zu liegen. Für eine mögliche Rückübertragung spricht die systemische Herangehensweise, die die Ergebnisse molekularer Analysen hinsichtlich des Gesamtorganismus einholen will. Mit Blick auf das Reduktandum bzw. den gesamtkontextualisierten Menschen werden aber auch gleich die Grenzen deutlich, insofern sich die Systembiologie noch im lebenswissenschaftlichen Kontext und den damit einhergehenden Problemen der Komplexität bewegt.

Derzeit vollzieht sich der dritte Schritt der Rückübertragung pharmakologischer Entwicklungen ${ }^{20}$ in den sozial-kulturellen Gesamtkontext als das Verfahren der Arzneimittelzulassung, indem Wirkstoffe für den allgemeinen Gebrauch freigegeben werden. Die Voraussetzungen zur Zulassung umfassen neben der pharmazeutischen Qualität und der Zusammenfassung der Merkmale des Arzneimittels vor allem dessen Wirksamkeit, Unbedenklichkeit sowie das Nutzen-Risiko-Verhältnis. ${ }^{21}$

Im Rahmen klinischer Studien fungiert die Good Clinical Practice als Leitfaden der Forschung und Prüfung. In der Verordnung über die Anwendung der Guten Klinischen Praxis bei der Durchführung von klinischen Prüfungen mit Arzneimitteln zur Anwendung am Menschen hat die Bundesregierung eine entsprechende EU-Richtlinie umgesetzt. Zweck dieser Verordnung ist die Einhaltung der guten klinischen Praxis, d. h. die Sicherheit und das Wohlergehen der betroffenen Personen und glaubwürdige Ergebnisse. Geregelt werden die "Aufgaben, Verantwortungsbereiche und Verfahren hinsichtlich der Planung, Genehmigung, Durchführung und Überwachung von klinischen Prüfungen am Menschen $«^{22}$ gemäß $\S 4$ (23) AMG: »Klinische Prüfung bei Menschen ist jede am Menschen durchgeführte Untersuchung, die dazu bestimmt ist, klinische oder pharmakologische Wirkungen von Arzneimitteln zu erforschen oder nachzuweisen oder Nebenwirkungen festzustellen oder die Resorption, die Verteilung, den Stoffwechsel oder die Ausscheidung zu untersuchen, mit dem Ziel, sich von der Unbedenklichkeit oder Wirksamkeit der Arzneimittel zu überzeugen. ${ }^{23}$

Die Pflichtangaben zum Prüfpräparat beziehen sich nach der GCP-Verordnung nur auf das materiale Präparat, d. h. Verfallsdatum, Dosierung etc. ${ }^{24}$ Dies gilt auch für die

19 Westerhoff 2007, S. 50.

20 Der Schritt von der Systembiologie zur Pharmazie, auch wenn es sich um zwei verschiedene Disziplinen handelt, ist hier insofern gangbar, als dass die Pharmazie auf (system)biologischen Grundlagen aufbaut.

21 Vgl. IQWiG 2011.

22 GCP-Verordnung $\$ 2$.

23 AMG $\$ 4(23)$.

24 Vgl. GCP-Verordnung $\$ 5$ Kennzeichnung von Prüfpräparaten. 
verfahrenstechnischen Details der Antragstellung. ${ }^{25}$ Eine weitere wichtige Rolle bei der Bewilligung spielen neben der Bundesoberbehörde die Ethik-Kommissionen als unabhängige Gremien, deren Aufgabe es ist, den Schutz der Rechte, die Sicherheit und das Wohlergehen betroffener Personen zu sichern und diesbezüglich Vertrauen in der Öffentlichkeit zu schaffen. Basierend auf der Deklaration von Helsinki ${ }^{26}$ liegen hier Kriterien wie die Effektivität, Effizienz, Verfügbarkeit und Qualität von Präparaten zugrunde. Bezüglich der Arbeit der Ethik-Kommissionen gilt es einschränkend anzumerken, dass neben dem nur geringen "Beurteilungsspielraum ${ }^{27}{ }^{27}$ sowie einer fehlenden Professionalisierung aufgrund der ehrenamtlichen Tätigkeit der Kommissionsmitglieder ${ }^{28}$ mit Blick auf die Komplexität menschlicher Lebenswelt vor allem die interdisziplinäre Besetzung problematisch ist, sofern nach EU-Recht lediglich die Disziplinen Gesundheitswesen und nicht-medizinische Bereiche unterschieden werden.

Auch die Bestimmung von Nebenwirkungen oder unerwünschten Ereignissen folgt lediglich einer vorwiegend pharmakologischen Logik. Das AMG formuliert: »Nebenwirkungen sind die beim bestimmungsgemäßen Gebrauch eines Arzneimittels auftretenden schädlichen unbeabsichtigten Reaktionen. Schwerwiegende Nebenwirkungen sind Nebenwirkungen, die tödlich oder lebensbedrohend sind, eine stationäre Behandlung oder Verlängerung einer stationären Behandlung erforderlich machen, zu bleibender oder schwerwiegender Behinderung, Invalidität, kongenitalen Anomalien oder Geburtsfehlern führen. ${ }^{29}$ Diese Liste ließe sich mit Blick auf unerwünschte Ereignisse und schwerwiegende unerwünschte Ereignisse in der gleichen Weise fortsetzen.

$\mathrm{Zu}$ guter Letzt spielt das Nutzen-Risiko-Verhältnis, zu dem auch die Kosten-/Nutzenrechnung zählt, eine herausragende Rolle. Das AMG definiert ein Risiko im Zusammenhang mit der Qualität, Sicherheit oder Wirksamkeit des Arzneimittels für die Gesundheit der Patienten und bezüglich unerwünschter Auswirkungen auf die Umwelt. Das Nutzen-Risiko-Verhältnis umfasst wiederum eine Bewertung der positiven therapeutischen Wirkungen des Arzneimittels im Verhältnis zu den möglichen Risiken.

Das im AMG genannte Nutzen-Risiko-Verhältnis impliziert die im Sozialgesetzbuch verankerte Kosten-Nutzen-Bewertung von Arzneimitteln, mit der vor allem die Erstattungsfähigkeit neuer Präparate festgestellt werden soll. Der Idee nach erhält das eigens zu diesem Zweck gegründete Institut für Qualität und Wirtschaftlichkeit im Gesundheitswesen in Köln einen Prüfauftrag vom Bundesgesundheitsministerium oder vom Gemeinsamen Bundesausschuss, in dem Leistungserbringer und Kostenträger sowie Patientenbeauftragte und Selbsthilfeorganisationen vertreten sind. Dabei soll nicht nur die relative Unschädlichkeit gegenüber einem Nutzen (ex negativo), sondern der positive Nutzen durch empirische Studien anhand von Kriterien des patientenrelevanten Nut-

\footnotetext{
Vgl. ebd. $\$ 7$.

Vgl. Weltärztebund $1964 f f$.

Pestalozza 2007, 177.

28 Vgl. ebd., 183.

29 AMG $\$ 4$ (13).
} 
zens nachgewiesen werden Diese lauten: Mortalität (Sterblichkeit/Sterberate), Morbidität (Krankheitshäufigkeit bezogen auf eine bestimmte Bevölkerungsgruppe), gesundheitsbezogene Lebensqualität sowie sekundär der interventionsbezogene Aufwand und die Patientenzufriedenheit. ${ }^{30}$ Einer nachhaltigen Umsetzung dieses erweiterten Prüfplanes stehen allerdings zwei substantielle Probleme entgegen. Zum einen fehlt eine gesetzlich verbindliche erweiterte Überprüfung des Nutzens vor der Markteinführung neuer Präparate. Eine weitere wichtige Forderung liegt in der konsequenten Veröffentlichung aller klinischen Studien, insbesondere negativer Ergebnisse, weil die Zulassungsanträge der Geheimhaltung unterliegen und die Pharmafirmen selbst entscheiden können, welche Studien veröffentlicht werden. ${ }^{31}$ In Anbetracht solcher Einschränkungen ließe sich die lebensweltliche Rückübertragung reduktiv gewonnener Forschungsresultate deutlich einfacher bewerkstelligen, wenn solche Hindernisse politischer Natur beseitigt würden. Damit ist die Rückübertragung nicht nur eine wissenschaftliche Aufgabe, sondern impliziert alle Akteure des Gesundheitssystems. Allerdings gibt es derzeit keine wahrnehmbaren Anzeichen einer Änderung, da trotz des Wissens um diese Möglichkeiten der politische Wille, pharmakologische Präparate einer kritischen Bewertung zu unterziehen, fehlt. Nicht nur die eher pharmafreundliche Neubesetzung des IQWiG, sondern auch schon die teils unzulängliche personelle Ausstattung der Ethik-Kommissionen sowie deren eingeschränkte Interventionsmöglichkeiten verhindern einen nachhaltigen Umgang mit den Resultaten hochtechnisierter Forschung. Die Arzneimittelzulassung verfährt also maßgeblich ex negativo, d. h. hinsichtlich der Feststellung einer relativen Unschädlichkeit eines Präparats gegenüber einem therapeutischen Nutzen. Erschwerend kommt »[e]ine Reduzierung des Instruments der Nutzenbewertung auf ein Hilfsmittel zur Preisbildung “ ${ }^{32}$ hinzu, so dass eine langfristige Nutzenbewertung, die erst nach der Einführung eines Präparats epidemologisch möglich ist, strukturell unterbunden wird. Ein eingeführtes Präparat oder eine Behandlungsmethode können nach ihrer Einführung und einer möglicherweise kritischen Nutzenbewertung schlicht am Markt bleiben. Die aktuellen Möglichkeiten einer verantwortungsvollen Rückübertragung lebenswissenschaftlicher Resultate in den lebensweltlichen Anwendungskontext sind somit insgesamt begrenzt, da die Kriterien zur Arzneimittelzulassung weitestgehend - mit Ausnahme des Nutzen-Risiko-Verhältnisses - dem reduktiven Rahmen der Lebenswissenschaften entstammen und sich nur auf die Markteinführung beschränken.

30 IQWiG 2011, S. 31ff. Ein alternatives Maß bilden sogenannte QALYs (quality adjusted life years), die den Nutzen einer medizinischen Intervention in einem einzigen Wert, dem Produkt aus Restlebenserwartung und Lebensqualität, wiedergeben; vgl. Deutscher Ethikrat 2011, S. $37 \mathrm{ff}$.

31 Vgl. dazu auch die entsprechenden Empfehlungen des Ethikrates in ebd., S. 94-97, sowie das Sondervotum.

32 Ebd., S. 61. 


\section{Ethische Implikationen}

In den bisher eingeschlagenen Wegen der lebensweltlichen Bewertung pharmazeutischer Präparate zeigen sich strukturell Kuhnsche Verluste, sofern die individuelle als auch die soziale Dimension der Konsumenten nicht angemessen berücksichtigt werden können. Dabei bietet die lebensweltliche Situierung genügend Kriterien für eine normative, $d$. h. letzthin lebensweltlich relevante Bewertung reduktionistisch gewonnener Präparate. Zu denken wäre hier an weiterführende Kriterien bezogen auf die individuelle Lebensqualität, die Einbeziehung der psychologischen Verfasstheit der Konsumenten mit Blick auch auf sogenannte Placeboeffekte sowie Langzeitevaluationen mit zulassungsrechtlich verbindlichen Resultaten. Insbesondere die Tatsache, dass pharmakologische Entwicklungen in einem hohen Maße gestaltbar sind, impliziert eine ethische Dimension und sollte eine zunächst kausalistisch fundierte Rückübertragung um lebensweltliche Kriterien ergänzen. ${ }^{33}$ Wenn es zwar auch nach dem derzeitigen Stand unmöglich erscheint, den instrumentellen Gebrauch molekularbiologischer Erklärungen systemisch zu kontextualisieren, so könnte methodologisch zumindest die Differenz zwischen Reduktans und Reduktandum berücksichtigt werden, um nicht sogleich einen Erfolg auf der Ebene des Reduktans auf das Reduktandum zu übertragen. Während die therapeutische Praxis eine solche Äquivokation zwingend verlangt, darf die Wissenslücke, die mit einer Übertragung und Instrumentalisierung reduktiv gewonnener Resultate einhergeht, nicht einseitig ökonomisch ausgenutzt werden. ${ }^{34}$ Eine methodologische Analyse kann aus den Randbedingungen zunächst weitere Bewertungskriterien ableiten, ${ }^{35}$ weil die Differenzen zwischen Reduktandum und Reduktans wissenschaftlich - sowohl methodologisch als auch disziplinär - genau benannt werden können. Demgegenüber muss eine politische und öffentliche Entscheidungsfindung dahin führen, wie die explanatorischen Lücken geschlossen bzw. welche Standards praktisch umgesetzt werden sollen. Aus wissenschaftstheoretischer Sicht lässt sich zusammenfassend festhalten, dass eine weitergehende Rückübertragung, als dies bisher geschieht, bei aller wissenschaftlichen Unkenntnis durchaus möglich wäre. Der Optimismus Freunds muss aber eben dahingehend eingeschränkt werden, dass nicht nur die Wahl des Zuganges durch den Wissenschaftler, sondern auch die öffentliche, politische und auch juristische Verwertung der Forschungsdaten und deren förderungspolitische Attraktivität ebenso konstituierende Faktoren darstellen. Die teils unbefriedigende Situation scheint daher zu rühren, dass die lebensweltliche Unvollständigkeit

33 Zum Begriff der Gestaltbarkeit vgl. Grunwald 2007.

$34 \mathrm{Vgl}$. insbesondere das Sondervotum in Deutscher Ethikrat 2011. Lübbe empfiehlt, das Kriterium der Kosteneffektivität »nicht als unabhängige[n] Bestandteil eines multikriterial gewichtenden Priorisierungskonzepts« (ebd., S. 122) zu veranschlagen.

35 Insbesondere das Verhältnis von Länge und Qualität des Lebens, wobei hier noch Methoden zu ent wickeln wären, die nicht nur wie die QALYs quantitativen und statistisch zumindest problematischen Messgrößen folgen. Zu denken wäre ebenso an weitere Anwendungsfelder als nur die Therapie. 
wissenschaftlicher Resultate billigend und aus strategischen Interessen in Kauf genommen wird, anstatt in der Rekontextualisierung fachwissenschaftlicher Forschung ein notwendiges Kriterium für die lebensweltliche Anwendung selbst zu sehen.

\section{Literatur}

Bringmann, Peter u. a.: Systems Biology. Applications and Perspectives. Berlin/Heidelberg 2007.

Bundesministerium der Justiz: Gesetz über den Verkehr mit Arzneimitteln (Arzneimittelgesetz$A M G$ ). 2005.

- , Verordnung über die Anwendung der Guten Klinischen Praxis bei der Durchführung von klinischen Prüfungen mit Arzneimitteln zur Anwendung am Menschen (GCP-Verordnung - GCP-V). 2006.

Cornish-Bowden, Athel: »Putting the Systems back into Systems Biology.«In: Perspectives in Biology and Medicine 49 (2006), H. 4, 475-489.

Deppert, Wolfgang u. a. (Hg.): Wissenschaftstheorien in der Medizin. Ein Symposium. Berlin/New York 1992.

Deutscher Ethikrat (Hg.): Nutzen und Kosten im Gesundheitswesen - Zur normativen Funktion ihrer Bewertung. Berlin 2011.

Folkers, Gerd u. a.: "Drug Design: Designer Drugs.«In: (Hg.) Konsorski-Lang, Silke - Hampe, Michael: The Design of Material, Organism, and Minds. Berlin/Heidelberg 2009, S. 53-64.

Forschungszentrum Jülich GmbH: Systembiologie. Ergebnisse, Fortschritte und Innovationen aus der BMBF-Förderung. Jülich/Berlin 2008.

Grüneberg, Patrick: »Die Ambivalenz zwischen Therapie und Leistung.»In: (Hg.) Asmuth, Christoph: Was ist Doping? Fakten und Probleme der aktuellen Diskussion. Bielefeld 2010, S. 117-137.

Grunwald, Armin: »Orientierungsbedarf, Zukunftswissen und Naturalismus. Das Beispiel der stechnischen Verbesserung، des Menschen.« In: Deutsche Zeitschrift für Philosophie 55 (2007), H. 6, 949-965.

Heinemann, Thomas: "Der menschliche Organismus aus Sicht der molekularen Medizin."In: (Hg.) Honnefelder, Ludger - Schmidt, Matthias C.: Naturalismus als Paradigma. Wie weit reicht die naturwissenschaftliche Erklärung des Menschen? Berlin 2007, S. 171-180.

Henning, Tim: »Naturalismus und erzählte Geschichte.«In: Allgemeine Zeitschrift für Philosophie 34 (2009), H. 2, 173-195.

Holzbach, Rüdiger u. a.: "Zusammenhang zwischen Verschreibungsverhalten der Ärzte und Medikamentenabhängigkeit ihrer Patienten.« In: Bundesgesundheitsblatt - Gesundheitsforschung Gesundheitsschutz 53 (2010), H. 4, 319-325.

Hoyningen-Huene, Paul - Wuketits, Franz M. (Hg.): Reductionism and systems theory in the life sciences: some problems and perspectives. Dordrecht u. a. 1989.

IQWiG: Allgemeine Methoden. Entwurf für Version 4.0 vom 09.03.2011. Köln 2011.

Pestalozza, Christian: „Ethik-Kommissionen und die klinische Prüfung von Arzneimitteln am Menschen aus deutscher Sicht.«In: HFR (2007), 177-191.

Rager, Günter: »Das Selbstverständnis der Medizin und die Grenzen der Naturalisierung: Medizin als praktische Wissenschaft."In: (Hg.) Honnefelder, Ludger - Schmidt, Matthias C.: Naturalismus als Paradigma. Wie weit reicht die naturwissenschaftliche Erklärung des Menschen? Berlin 2007, S. 348-361. 
Rosen, Robert: „Die Philosophie des `Handwerklichen` - Ein Beitrag zur Frage der Beschreibung und Handhabung komplexer Systeme.« In: (Hg.) Deppert, Wolfgang u. a.: Wissenschaftstheorien in der Medizin. Ein Symposium. Berlin/New York 1992, S. 259-274.

Schneider, Silvia u. a.: DIPS. Berlin/Heidelberg 2011, S. 177-194.

Schumann, Heiko: Die Kostenentwicklung im Deutschen Gesundheitswesen: Im Blickpunkt: Der erste und zweite Gesundheitsmarkt unter dem Einfluss des demographischen Wandels, medizinisch technischen Fortschritts und Moral Hazard. München 2011.

Sellars, Wilfrid: »Empiricism and the Philosophy of Mind.«In: (Hg.) Ders.: Science, Perception, and Reality. London 1963, S. 127-196.

Stöckler, Manfred: "Plädoyer für einen pragmatisch eingeschränkten Reduktionismus.« In: (Hg.) Deppert, Wolfgang u. a.: Wissenschaftstheorien in der Medizin. Ein Symposium. Berlin/New York 1992, S. 157-182.

Tian, Qiang u. a.: »Systems cancer medicine: towards realization of predictive, preventive, personalized and participatory (P4) medicine.« In: Journal of internal medicine 271 (2012), H. 2, 111121.

Trewavas, Anthony: »A brief history of systems biology.«In: The Plant Cell 18 (2006), H. 10, 24202430.

Vollmer, Gerhard: „Was ist Naturalismus?« In: (Hg.) Keil, Geert - Schnädelbach, Herbert: Naturalismus. Philosophische Beiträge. Frankfurt a. M. 2000, S. 46-67.

Weltärztebund: Deklaration von Helsinki: Ethische Grundsätze für die medizinische Forschung am Menschen. Helsinki 1964ff.

Westerhoff, Hans V.: "Systems Biology: New Paradigms for Cell Biology and Drug Design." In: (Hg.) Bringmann, Peter u. a.: Systems Biology. Applications and Perspectives. Berlin/Heidelberg 2007, S. 45-67.

Wolters, Gereon: »Reduktionismus.«In: (Hg.) Mittelstraß, Jürgen: Enzyklopädie Philosophie und Wissenschaftstheorie. Bd. 1, Stuttgart/Weimar 1996, S. 521-522. 